\title{
Possible Manifestations of the Chiral Anomaly and Evidence for a Magnetic Field Induced Topological Phase Transition in the Type-I Weyl Semimetal TaAs
}

\author{
Q. R. Zhang \\ B. Zeng \\ Y. C. Chiu \\ R. Schönemann \\ S. Memaran
}

See next page for additional authors

Follow this and additional works at: https://bearworks.missouristate.edu/articles-cnas

\section{Recommended Citation}

Zhang, Q. R., B. Zeng, Y. C. Chiu, R. Schönemann, S. Memaran, W. Zheng, D. Rhodes et al. "Possible manifestations of the chiral anomaly and evidence for a magnetic field induced topological phase transition in the type-I Weyl semimetal TaAs." Physical Review B 100, no. 11 (2019): 115138.

This article or document was made available through BearWorks, the institutional repository of Missouri State University. The work contained in it may be protected by copyright and require permission of the copyright holder for reuse or redistribution.

For more information, please contact BearWorks@library.missouristate.edu. 


\section{Authors}

Q. R. Zhang; B. Zeng; Y. C. Chiu; R. Schönemann; S. Memaran; W. Zheng; D. Rhodes; K.-W. Chen; Tiglet Besara; and For complete list of authors, see publisher's website. 


\title{
Possible manifestations of the chiral anomaly and evidence for a magnetic field induced topological phase transition in the type-I Weyl semimetal TaAs
}

\author{
Q. R. Zhang, ${ }^{1,2}$ B. Zeng, ${ }^{1}$ Y. C. Chiu, ${ }^{1,2}$ R. Schönemann, ${ }^{1}$ S. Memaran, ${ }^{1,2}$ W. Zheng, ${ }^{1,2}$ D. Rhodes, ${ }^{1,2}$ K.-W. Chen, ${ }^{1,2}$ \\ T. Besara, ${ }^{1}$ R. Sankar, ${ }^{3}$ F. Chou, ${ }^{3}$ G. T. McCandless, ${ }^{4}$ J. Y. Chan, ${ }^{4}$ N. Alidoust, ${ }^{5}$ S.-Y. Xu, ${ }^{5}$ \\ I. Belopolski, ${ }^{5}$ M. Z. Hasan, ${ }^{5}$ F. F. Balakirev, ${ }^{6}$ and L. Balicas $\mathbb{1}^{1,2}$ \\ ${ }^{1}$ National High Magnetic Field Laboratory, Florida State University, Tallahassee, Florida 32310, USA \\ ${ }^{2}$ Department of Physics, Florida State University, Tallahassee, Florida 32306, USA \\ ${ }^{3}$ Center for Condensed Matter Sciences, National Taiwan University, Taipei 10617, Taiwan \\ ${ }^{4}$ University of Texas at Dallas, Department of Chemistry and Biochemistry, Richardson, Texas 75080, USA \\ ${ }^{5}$ Laboratory for Topological Quantum Matter (B7), Department of Physics, Princeton University, Princeton, New Jersey 08544, USA \\ ${ }^{6}$ National High Magnetic Field Laboratory, Los Alamos National Laboratory, MS E536, Los Alamos, New Mexico 87545, USA
}

(Received 30 November 2018; revised manuscript received 5 September 2019; published 17 September 2019)

\begin{abstract}
We studied the magnetoresistivity and the Hall effect of the type-I Weyl semimetal TaAs to address the controversy surrounding its anomalous transport properties in relation to its bulk topological character. For fields and currents along the basal plane, we observe a very pronounced planar Hall effect (PHE) upon field rotation with respect to the crystallographic axes at temperatures as high as $T=100 \mathrm{~K}$. Parametric plots of the PHE signal as a function of the longitudinal magnetoresistivity (LMR) collected at $T=10 \mathrm{~K}$ lead to concentric traces as reported for $\mathrm{Na}_{3} \mathrm{Bi}$ and GdBiPt. This would suggest that the negative LMR and the PHE observed in TaAs are intrinsically associated with the axial anomaly among its Weyl nodes. For fields nearly along the $a$ axis we observe hysteresis as one surpasses the quantum limit, where the magnetic torque indicates a change in regime as the field increases, i.e., from paramagnetism and diamagnetism due to Weyl fermions above and below the Weyl node(s), respectively, to a paramagnetic one associated with the field-independent $n=0$ Landau level. Hysteresis coupled to the overall behavior of the torque would be consistent with a topological phase transition associated with the suppression of the Weyl dispersion at the quantum limit. This transition leads to the suppression of the negative LMR confirming that it is intrinsically associated with the Weyl dispersion. The Hall effect for fields along the $c$ axis reveals two successive changes in slope, or two successive decrements in carrier mobility, one at the quantum limit and a second one at the critical field where a phase transition toward an insulating state was recently reported. This suggests the possibility of two successive phase transitions as function of the field with the higher-field one involving solely the $n=0$ Landau level. Finally, for both field orientations we observe Shubnikov-de Haas like oscillations beyond the quantum limit hence involving quasiparticles at fractional filling factors.
\end{abstract}

DOI: 10.1103/PhysRevB.100.115138

\section{INTRODUCTION}

Weyl semimetals, like the Ta and $\mathrm{Nb}$ based monopnictides, are characterized by a lack of inversion symmetry, strong spinorbit coupling, and linearly dispersing bands in three dimensions which are modeled via two copies of the Weyl equation [1-4]. The Weyl equation [5] was originally proposed as an alternative to the Dirac one [6] for describing spin-1/2 massless particles, or neutrinos. Band structure calculations [2,3] and angle-resolved photoemission experiments find that these compounds are characterized by crossings between valence and conduction bands at specific points in the Brillouin zone around which the bands disperse linearly as in Dirac systems [2,7-11]. Theoretical calculations indicate that these so-called Weyl points occur in pairs, are topologically nontrivial, and act as either sinks or sources of Berry curvature, thus acting as topological charges [2,3]. Therefore, charge carriers undergoing electronic orbits around one, or in between a pair, of these points would acquire a net chirality in their velocity and hence a concomitant Berry phase, thus effectively acting as Weyl fermions. The calculations [2,3] predict the existence of 12 pairs of Weyl points in the first Brillouin zone of the $\mathrm{Nb}$ and $\mathrm{Ta}$ monopnictides, 4 pairs at $k_{z}=0$, and 8 additional ones at $k_{z} \sim$ $0.59 \pi$. Each pair is composed of Weyl points with opposite chirality or opposite topological charge. The projection of the band structure toward the surface of these materials leads to topologically nontrivial surface states, namely Fermi arcs connecting points on the surface which are the projection of the bulk Weyl points [2,3,7-12]. The separation between these points, and hence the length of the Fermi arcs, is found to increase with the strength of the spin-orbit coupling [12].

The application of an external magnetic field is predicted to break the chiral symmetry between Weyl fermions [13-17,19]. This leads to the so-called Adler-Bell-Jackiw or axial anomaly [13-17,19], which corresponds to a net flow of Weyl fermions along the axis connecting Weyl points of opposite chirality. For clean systems, and depending on the functional form of the quasiparticle scattering potential [16], this "axial" current is predicted $[15,16]$ to induce a 
net increase in the longitudinal magnetoconductivity (when $\mu_{0} \vec{H} \| \vec{j}$, where $j$ is the current density). This effect was claimed to have been observed in TaAs [17-19] although more recently it was argued to be an artifact resulting from current inhomogeneity or "current jetting" [20,21]. The chiral anomaly is predicted to lead to novel effects, such as a novel type of quantum oscillatory phenomenon in very thin crystals, i.e., cyclotron orbits traversing the bulk of the crystal (along the smallest dimension) and involving the Fermi arcs at the opposite surfaces of the crystal [22,23], or to a planar Hall effect for fields rotating within the plane of the electrical current $[24,25]$. Elucidating the role of the chiral or axial anomaly and exposing its related, novel optoelectronic properties is a relevant subject in condensed matter physics and the leading motivation for this study.

Since the Fermi surfaces (FSs) of semimetallic systems, in particular those of the monopnictides, are rather small $[19,20,23,26-29]$, it is possible to reach the quantum limit (QL) with available magnetic fields. In its conventional definition, the quantum limit is reached when the energy of all Landau levels, with the exception of the $n=0$ level, exceeds that of the Fermi level according to the following dispersion relations:

$$
\begin{gathered}
E\left(n, k_{z}\right)=\left(n+\frac{1}{2}\right) \frac{\hbar e B}{\mu}+\frac{\hbar^{2} k_{z}^{2}}{2 \mu} \\
=\hbar v_{F} \sqrt{2 B(n+\gamma)+k_{z}^{2}},
\end{gathered}
$$

where Eqs. (1) and (2) describe the electronic dispersions for conventional and Weyl-like carriers in metallic systems, respectively. Here, $e$ corresponds to the electron charge, $\hbar$ to the Planck constant, $v_{F}$ is the Fermi velocity, $\mu$ the carrier effective mass, $B$ the magnetic induction field, and $\gamma$ the phase of the charge carrier ( $\gamma=0$ for Weyl and Dirac systems). It turns out that a sharp anomaly, or an abrupt change in the sign of the magnetic torque, was observed upon approaching the QL in NbAs [26]. This observation was attributed to a change in regime, from a region dominated by competing magnetic interactions, i.e., paramagnetism and diamagnetism for Weyl fermions above and below the Weyl node(s), respectively, to a paramagnetic one associated with the field-independent $n=0$ Landau level [26]. There are also reports of anomalies in the longitudinal magnetoresistivity of TaAs which are observed well beyond the QL for magnetic fields applied along its $c$ axis [17]. These anomalies were suggested to result from electronic interactions, or a nesting instability among Weyl electrons possibly leading to helical spin-density waves [17]. Electronic instabilities, proposed to be magnetic-fieldinduced charge-density waves associated with the quasi-onedimensional electronic dispersion described by Eq. (1), are indeed observed in graphite beyond its QL [30].

According to Ref. [21], due to current jetting, it would be possible to expose the effect of the chiral anomaly on the transport properties only for Weyl systems characterized by relatively low mobilities and by rather small values of the quantum limit field $\mu_{0} H_{Q}$. Examples would include $\mathrm{Na}_{3} \mathrm{Bi}$ [31] and GdBiPt [21] whose $\mu_{0} H_{Q}$ is on the order of just 5-6 T. Surprisingly, Ref. [21] observes a net increase in the magnetoconductivity for both systems, ascribed to the chiral anomaly, for fields all the way up to $\mu_{0} H>2 \mu_{0} H_{Q}$, values that are well beyond the last occupied Landau level in the Weyl dispersion. Therefore, the interplay between the chiral anomaly and the QL remains poorly understood.

Here, we evaluate in detail the magnetotransport properties of TaAs as well as its bulk electronic structure at the Fermi level, through measurements at low temperatures and magnetic fields surpassing its quantum limit. Independently of field orientation, and for every sample measured, we observe a pronounced asymmetry between the magnetoresistivity collected under positive and negative fields due to a very pronounced superimposed Hall-like signal. Analysis of the current distribution following Ref. [21] for fields parallel to the electrical currents flowing along the basal plane yields results that are inconsistent with the current jetting scenario. At lower temperatures, the negative magnetoresistivity initially claimed to result from the chiral anomaly among Weyl nodes is observed in every measured crystal. We also observe a pronounced planar Hall effect whose amplitude is considerably larger than the anisotropy of the magnetoresistivity, when the field is rotated within the plane of the current. Parametric plots of the angular dependence of the planar Hall signal as a function of the angle-dependent longitudinal magnetoresistivity lead to concentric traces which, according to Ref. [21], would indicate that both quantities are dominated by the Adler-Bell-Jackiw anomaly. The negative magnetoresistivity survives until the quantum limit is reached, where a hysteretic topological/electronic phase transition observed to occur indicating that it is intrinsically associated with the original Weyl dispersion. We conclude that these observations support previous experimental claims and theoretical predictions proposing a primordial role for the axial anomaly in monoarsenides that is suppressed once the quantum limit is reached.

\section{RESULTS}

Single crystals of monoarsenides were grown via a chemical vapor transport technique, as previously described [32]. Polycrystalline precursor specimens were first prepared by sealing elemental Ta and As mixtures under vacuum in quartz ampoules and by heating the mixtures at a rate of $100^{\circ} \mathrm{C} / \mathrm{h}$ to $700^{\circ} \mathrm{C}$, and kept at this temperature for 3 days. The polycrystalline TaAs boules were subsequently sealed under vacuum in quartz ampoules with iodine to serve as the transporting agent. The ampoules were slowly heated up in a horizontal tube furnace under a temperature gradient $\Delta T=100^{\circ} \mathrm{C}$. The ampoules were maintained under this condition for 3 weeks before rapidly cooling them down to room temperature. This process produced a large number of single crystals with typical dimensions of $0.5 \mathrm{~mm}^{3}$.

The crystallographic axes of the measured single crystals were identified through single-crystal $\mathrm{x}$-ray diffraction and subsequently polished to produce bar-shaped crystals with their longer axes along the basal planes. Torque magnetometry was measured using a $\mathrm{CuBe}$ cantilever beam technique whose deflection was measured capacitively. Electrical contacts were produced by attaching gold wires with silver paint in a standard four-terminal configuration for either magnetoresistivity or Hall effect measurements. Transport measurements were 
performed via a conventional AC technique in either a physical properties measurement system, a $35 \mathrm{~T}$ resistive magnet, or the $45 \mathrm{~T}$ hybrid magnet at the National High Magnetic Field Laboratory (NHMFL) in Tallahassee in combination with either a ${ }^{3} \mathrm{He}$ cryostat or variable temperature insert. Measurements were also performed in a $60 \mathrm{~T}$ pulse field magnet at the NHMFL at Los Alamos National Laboratory. Throughout this paper longitudinal magnetoresistivity $\rho_{x x}^{L}$ refers to $\vec{j} \| \mu_{0} \vec{H}$ while transverse magnetoresistivity $\rho_{x x}^{T}$ refers to $\vec{j} \perp \mu_{0} \vec{H}$, where $\vec{j}$ is the current density.

\section{A. Evaluation of the electrical current distribution}

The present debate on the electrical transport properties of the TaAs family of compounds is focused on the origin of the observed negative longitudinal magnetoresistivity, or on whether it results from the so-called current jetting effect $[20,21]$ or from an increase in magnetoconductivity associated with the emergence of an axial current between Weyl points [13-17,19]. In this respect, Ref. [21] proposed a few experimental tests to evaluate the current inhomogeneity in samples characterized by high carrier mobilities. For instance, Ref. [21] proposed the use of point contacts for both current and voltage leads demonstrating for elemental $\mathrm{Bi}$ that in a situation dominated by current jetting, one would measure positive magnetoresistivity $\left[\partial \rho_{x x}^{L} / \partial\left(\mu_{0} H\right)>0\right.$ ] through voltage leads placed along a line (or along the "spine" of the crystal) connecting the current contacts. Instead, a pronounced negative one $\left[\partial \rho_{x x}^{L} / \partial\left(\mu_{0} H\right)<0\right]$ is observed for leads placed at the lateral edge of the crystal due to a magnetic-fieldinduced current redistribution. In $\mathrm{Bi}$, and for this combination of field and current orientation(s), this behavior was described by Yoshida [33] who found that the field concentrates the current density toward the middle of the crystal.

Following the methodology in Ref. [21], Fig. 1(a) displays $\rho_{x x}^{L}$ for a TaAs crystal or sample 1 normalized with respect to its maximum value $\rho_{\max }^{L}$ as a function of positive and negative magnetic fields and for both configurations of electrical contacts (see sketch to the right). In the sketch $V_{\mathrm{S}}$ and $V_{\mathrm{E}}$ correspond to the voltages collected through the contacts placed along the spine and the edge of the crystal, respectively. This crystal, as well as all the other crystals studied here, was oriented through single-crystal x-ray diffraction and polished to reduce its thickness along the $c$ axis resulting, in this case, in a relatively thick platelet having a thickness $t \simeq 0.3 \mathrm{~mm}$. Crystals measured at high fields were also polished to similar thicknesses. Here, we chose conventional current contacts instead of pointlike ones to evaluate the current inhomogeneity affecting our subsequent measurements performed through a four-terminal configuration. As shown in Ref. [35] for elemental $\mathrm{Cr}$ crystals, conventional current leads do not prevent the simultaneous observation of positive and negative magnetoresistivities among different pairs of voltage leads as seen in Refs. [20] and [21] for pointlike contacts.

As previously reported in Ref. [20] (see the Supplemental Material therein), one observes a remarkable asymmetry between traces collected at positive and negative fields which will be reanalyzed below. In addition, $\rho_{x x}^{L}$ is observed to display a sharp increase at low fields akin to the one reported by Pippard [36] for elemental metals, but instead of saturating

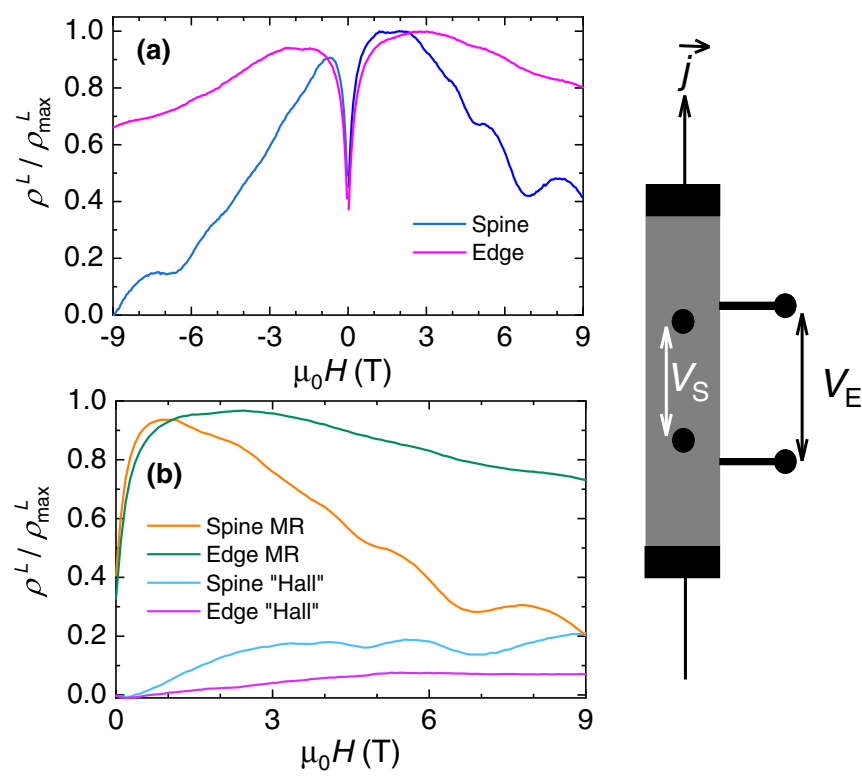

FIG. 1. Role of current distribution on the longitudinal magnetoresistivity. (a) Raw longitudinal magnetoresistivity $\rho^{L}$, i.e., for currents nearly aligned along the magnet field $\mu_{0} H$, normalized by its maximum value $\rho_{\max }^{L}$ for a TaAs crystal (crystal 1 ) as a function of the field $\mu_{0} H$ and for currents and fields along the $a$ axis of the crystal. We used point contacts at one edge of the sample (magenta line) and along its "spine" (blue line) to measure the respective voltages $V_{\mathrm{E}}$ and $V_{\mathrm{S}}$, as illustrated in the side sketch, following the method prescribed in Ref. [21] to analyze current jetting. Negative magnetoresistivity, or $\partial \rho^{L} / \partial\left(\mu_{0} H\right)<1$, is always observed and is more pronounced for the contacts along the spine of the crystal. Notice the marked asymmetry in $\rho^{L}\left(\mu_{0} H\right)$, or Hall-like signal, between traces collected under positive and negative fields. This asymmetry is observed in every crystal and for the different configuration of contacts, as discussed in the main text. (b) Average of the magnetoresistivity (or MR) for both field orientations and for the contacts along the spine (orange) and at the edge (green trace) of the crystal. The same panel contains also the asymmetric component, or the difference between positive and negative field traces for contacts along the spine (magenta) and at the edge (clear blue) of the crystal.

at higher fields it is followed by the previously reported negative longitudinal magnetoresistivity (LMR) $[17,19]$. The negative LMR is far more pronounced for the contacts placed along the spine of the crystal when compared to the one measured through the contacts placed at its edge. This is the exact opposite of the behavior reported in Refs. [20,21,35] and of the theoretical predictions in Ref. [33], strongly suggesting that current jetting does not dominate the longitudinal transport in this crystal. Instead, the consistent observation of negative magnetoresistivity, particularly in $V_{\mathrm{S}}$ or regardless of the precise placement of the voltage leads, suggests that the negative LMR is intrinsic to TaAs. This will be confirmed through an additional test proposed in Ref. [21].

Figure 1(b) displays the average (or "MR") and the difference (or "Hall") between traces collected under both field orientations and for the voltage contacts along the spine and the edge of the sample. As seen, the averages decrease as the field increases, while the differences behave as Hall-like signals: starting from zero and saturating as the field increases, 

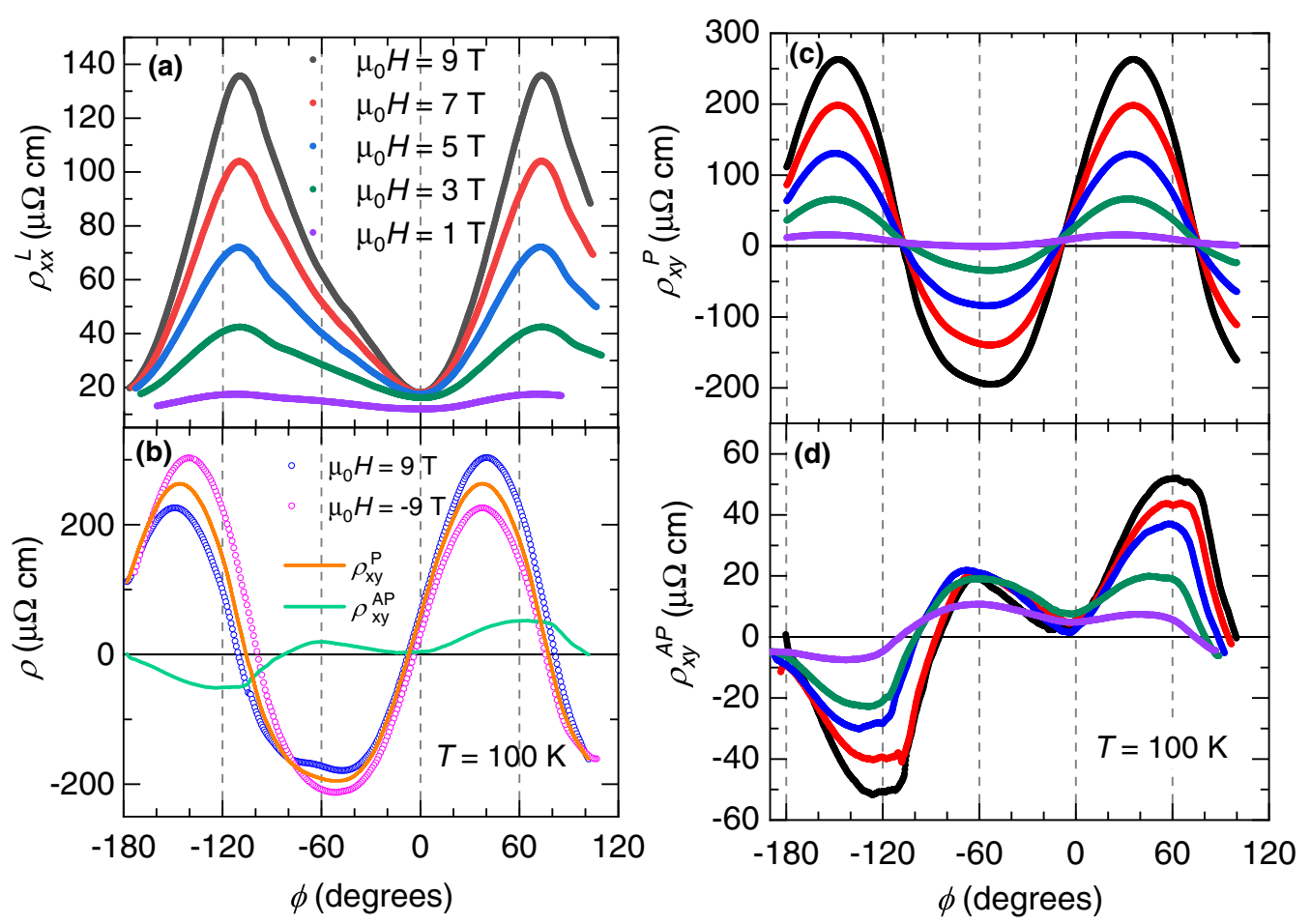

FIG. 2. Angular dependence of the planar magnetoresistivity and observation of the planar Hall effect at $T=100 \mathrm{~K}$. (a) Angular dependence of the magnetoresistivity $\rho_{x x}^{L}$ (measured via a conventional four-terminal configuration of contacts) for several values of the field $\mu_{0} \mathrm{H}$ at a temperature $T=100 \mathrm{~K}$. These curves correspond to the average between angular sweeps collected at positive and negative fields. $\phi$ is the planar angle between the electrical current $\vec{j}$ injected along the $a$ axis and $\mu_{0} H$. Over the entire angular range $\rho_{x x}^{L}$ increases as $\mu_{0} H$ increases. (b) Planar Hall signal measured under $\mu_{0} H=9 \mathrm{~T}$ via a four-terminal Hall configuration of electrical contacts and for both field orientations. The angular asymmetry between both traces or $\rho_{x y}^{A P}$ (green trace) contrasts with the symmetric component $\rho_{x y}^{P}$ (orange trace) resulting from the average of $\rho_{x x}^{L}$ with respect to both field orientations. $\rho_{x y}^{A P}(\phi)$ displays a periodicity of $360^{\circ}$ while $\rho_{x y}^{P}(\phi)$ is periodic over $180^{\circ}$. (c) $\rho_{x y}^{P}$ as a function of $\phi$ at $T=100 \mathrm{~K}$ and for several fields. The amplitude $\rho_{x y}^{P}(\phi)$ is considerably larger than that of $\rho_{x x}^{L}(\phi)$ implying that it does not result from the anisotropy of the magnetoresistivity. (d) Asymmetric component superimposed onto the raw planar Hall signal or $\rho_{x y}^{A P}$ at $T=100 \mathrm{~K}$ as a function of $\phi$ for several fields. These curves correspond to the difference between angular sweeps collected at positive and negative field values. Panels (b), (c), and (d) use the same marker color scheme to identify applied magnetic field values; namely, black, red, blue, green, and violet markers correspond to $\mu_{0} H=9,7,5,3$, and $1 \mathrm{~T}$, respectively.

with the nonlinearity being the hallmark of the Hall effect in a two-carrier system.

\section{B. Electrical transport properties as a function of magnetic field orientation}

Now we proceed with the additional tests proposed in Ref. [21] through the study of the angular dependence of the transport properties of TaAs with respect to magnetic field orientation. Figure 2 shows four-terminal resistivity $\rho\left(\mu_{0} H, \phi\right)$ for $\mu_{0} H \perp c$-axis and as a function of the angle $\phi$, using conventional configurations for the electrical contacts, as opposed to pointlike contacts, to measure either the magnetoresistivity or the planar Hall effect. For these measurements, the sample remained attached onto the rotator while only the voltage leads were silver painted, subsequently removed, and silver painted again in a different configuration. Here, $\phi$ is the angle between the current density $\vec{j}$ and $\mu_{0} \vec{H}$ applied along the basal plane, where $\phi=0^{\circ}$ corresponds to $\mu_{0} H \| j$. Figure 2(a) shows the magnetoresistivity $\rho_{x x}^{L}(\phi)$ traces collected at $T=100 \mathrm{~K}$ under several field values. As will be further illustrated below, one still observes a sizable asymmetry between traces collected under positive and negative fields and, therefore, these traces correspond to the average of the data collected under both field orientations. At $T=100 \mathrm{~K} \rho_{x x}^{L}(\phi)$ always increases with field regardless of its orientation. $\rho_{x x}^{L}(\phi)$ displays a $180^{\circ}$ periodicity following a sawtooth instead of a simple sinusoidal function as one would expect for a system driven from zero to maximum Lorentz force upon rotation, perhaps due to the sample's geometry. Figure 2(b) displays raw resistivity as a function of $\phi$ for traces collected under $\mu_{0} H=+9 \mathrm{~T}$ (magenta markers) and $-9 \mathrm{~T}$ (blue makers) through a conventional Hall-like configuration for the electrical contacts. Notice again the marked asymmetry between both traces. Therefore, the orange markers depict the average between both traces, or the planar Hall signal $\rho_{x y}^{P}$, having maxima with the exact same amplitude at either side of the minimum. The planar Hall effect (PHE) was observed recently in GdPtBi [37] and in $\mathrm{Cd}_{3} \mathrm{As}_{2}$ [38], and proposed to result from the axial anomaly between Weyl points [24,25]. It is observed in ferromagnetic metals and in semiconductors subjected to strong spin-orbit coupling and interpreted to result from the anisotropy in the magnetoresistivity induced by the inherent magnetic anisotropy of the system. In nonmagnetic Weyl systems, the chiral anomaly is predicted to induce a giant PHE [24,25], or the appearance of a large 

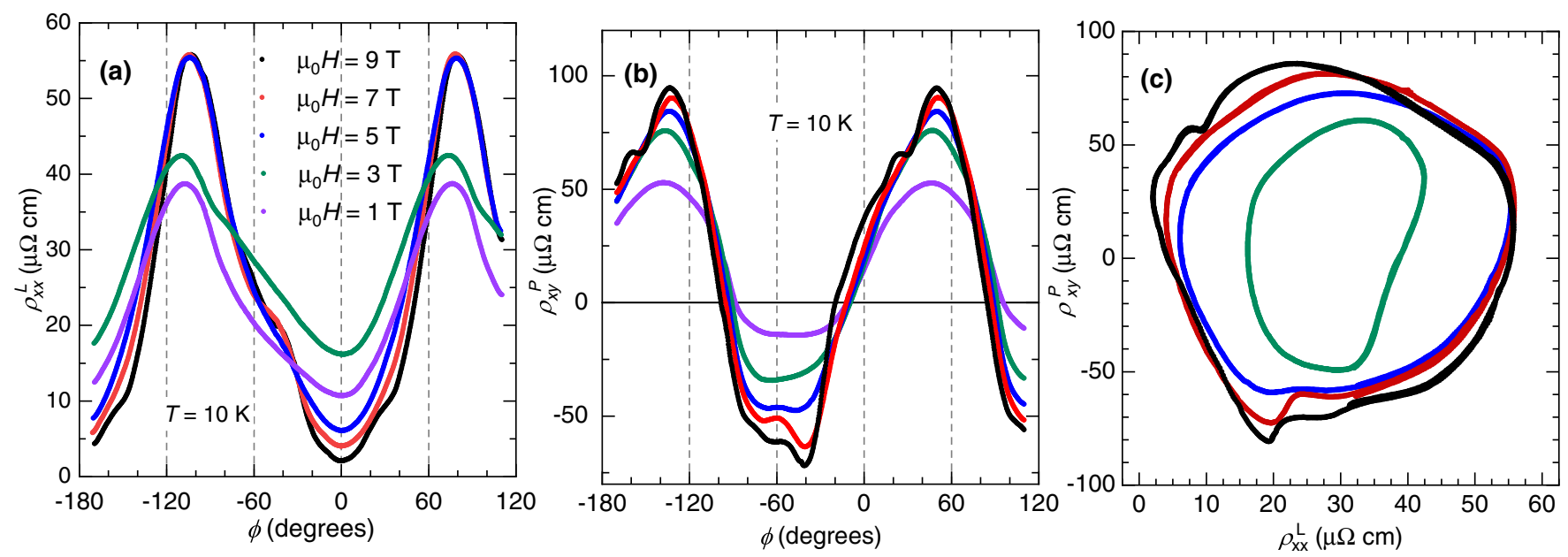

FIG. 3. Planar Hall effect and parametric plots at $T=10 \mathrm{~K}$. (a) Angular dependence of the planar magnetoresistivity $\rho_{x x}^{L}$ measured through the four-terminal configuration of contacts at a temperature $T=10 \mathrm{~K}$ and for several field values. For a given field value, these curves correspond to the average between angular sweeps collected under both field orientations. In contrast to the data collected at $T=100 \mathrm{~K}, \rho_{x x}^{L}$ is found to decrease with increasing field for $|\phi| \lesssim\left|60^{\circ}\right|$ while it increases with field for $|\phi| \gtrsim\left|60^{\circ}\right|$. (b) Symmetric component $\rho_{x y}^{P}$, or the average between the $\rho$ traces collected under both field orientations when measured through a conventional Hall-like configuration of contacts. These traces depict the planar Hall effect albeit with the superimposed Shubnikov-de Haas effect. (c) Parametric plots of $\rho_{x y}^{P}$ as a function of $\rho_{x x}^{P}$ at $T=10 \mathrm{~K}$ for several fields. Notice that these traces are nearly concentric which, according to Ref. [21], is a clear indication for the axial anomaly among Weyl nodes leading to the negative magnetoresistivity observed in TaAs. Panels (a), (b), and (c) use the same color scheme to identify the field values, namely, black, red, blue, green, and violet markers for $\mu_{0} H=9,7,5,3$, and $1 \mathrm{~T}$, respectively.

transverse voltage leading to $\rho_{x y} \propto \sin (2 \phi)$, as seen in in Fig. 2(c) which displays $\rho_{x y}^{P}$ as a function of $\phi$ for several field values. Its periodicity, i.e., $\sim 180^{\circ}$, coincides with previous reports on GdPtBi [37] and on $\mathrm{Cd}_{3} \mathrm{As}_{2}$ [38] and would indicate Weyl cones displaying no tilting with respect to the Fermi level [25]. The PHE has also been observed in $T_{d}-\mathrm{MoTe}_{2}$, see Ref. [39], and in $\mathrm{NiTe}_{2}$ (Ref. [40]) but in both cases it was claimed to result from the anisotropy of the its magnetoresistivity given the nonobservation of the negative longitudinal magnetoresistivity that otherwise would support a role for the axial anomaly. In the case of $T_{d}-\mathrm{MoTe}_{2}$ the amplitude of PHE signal is found to be nearly identical to the anisotropy of its magnetoresistivity [39], in contrast to what is seen here whose amplitude nearly doubles the maximum value shown by the planar magnetoresistivity. In fact, for any given field value the amplitude of the planar Hall signal is considerably larger than the anisotropy of the magnetoresistivity pointing to an unconventional origin - likely the chiral anomaly as proposed by Refs. [24] and [25].

The difference between traces collected under both field orientations yields another periodic signal, the asymmetric planar Hall $\rho_{x y}^{A P}$ (green markers) that has a much smaller amplitude than the PHE. For this experimental configuration, one should not observe any Hall-like signal under normal circumstances, albeit it is possible that it results from a small misalignment between the current and the external field. This asymmetric signal is odd in angle as the PHE and as a true Hall effect it is an odd function of the field and, therefore, akin to the anomalous planar Hall effect (APHE) recently reported [41] for $\mathrm{ZrTe}_{5}$. According to the authors of Ref. [41], the close correlation between the temperatures where the negative LMR and the APHE are observed indicates that the Weyl nodes and related Berry-phase texture would be responsible for this APHE. Its periodicity cannot be easily attributed to sample misalignment, or to a component of the field along the $c$ axis, since it should lead to a single sinusoidal function instead of the complex oscillatory pattern. This anomalous Hall signal displays a distinct temperature dependence with respect to the conventional transverse Hall effect shown below: if one assumed that it results from sample misalignment, one would obtain a misalignment angle of about $\sim 2.5^{\circ}$ at $T=5 \mathrm{~K}$, that would increase up to nearly $\sim 23^{\circ}$ at $T=100 \mathrm{~K}$. Here, we merely expose its observation suggesting that a more comprehensive study is required. Figure 2(d) displays $\rho_{x y}^{A P}$ as a function of $\phi$ for several field values.

\section{Parametric plots}

Figure 3 provides a similar evaluation of the angular dependence of the transport properties of TaAs but under $T=10 \mathrm{~K}$. Figure 3(a) displays the symmetrized $\rho_{x x}^{L}$ as a function of $\phi$ at $T=10 \mathrm{~K}$. In contrast to its behavior at $T=100 \mathrm{~K}$, within a broad angular range, i.e., $|\phi| \lesssim\left|60^{\circ}\right|, \rho_{x x}^{L}(\phi)$ decreases as the field increases, as previously reported [17,19]. Despite the suppression of phonon scattering, and for reasons to be determined, the overall amplitude of $\rho_{x x}^{L}(\phi, T=10 \mathrm{~K})$ decreases considerably with respect to the traces collected at $T=100 \mathrm{~K}$. Figure 3(b) displays the PHE signal $\rho_{x y}^{P}$ collected at $10 \mathrm{~K}$ as a function of $\phi$. Similarly to $\rho_{x x}^{L}$, the amplitude of $\rho_{x y}^{P}$ at $T=10 \mathrm{~K}$ becomes considerably smaller. In Figs. 3(a) and 3(b), an important difference with respect to the traces collected at $T=100 \mathrm{~K}$ is the superposition of the Shubnikovde Haas effect whose oscillatory pattern deforms the original wave form observed at that temperature. Figure 3(c) corresponds to the parametric plots of $\rho_{x y}^{P}(\phi)$ as a function of $\rho_{x x}^{L}(\phi)$ which, according to Ref. [21], can clarify whether the negative magnetoresistivity is indeed ascribable to the chiral anomaly as claimed for $\mathrm{Na}_{3} \mathrm{Bi}$ [31] and GdBiPt [21] or results 

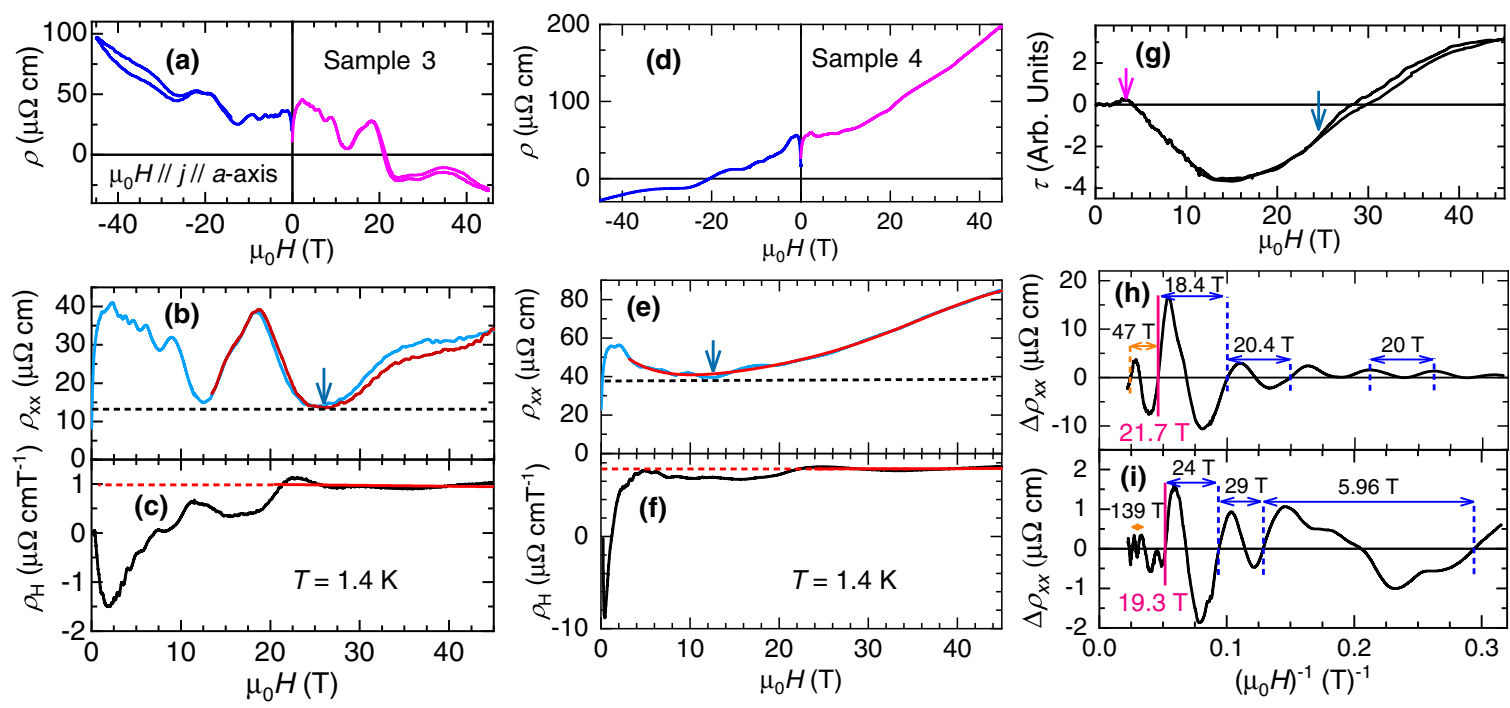

FIG. 4. Suppression of the negative longitudinal magnetoresistivity at a phase transition upon surpassing the quantum limit. (a) Raw resistivity $\rho$ for a third TaAs single crystal (sample 3) as a function of $\mu_{0} H \| a$-axis at $T=1.4 \mathrm{~K} . \rho\left(\mu_{0} H\right)$ displays a marked asymmetry with respect to both field orientations, indicating the superposition of a Hall-like component. (b) Averaged resistivity, or $\rho_{x x}=\left[\rho\left(\mu_{0} H\right)+\right.$ $\left.\rho\left(-\mu_{0} H\right)\right] / 2$, as a function of $\mu_{0} H . \rho_{x x}$ decreases beyond $\mu_{0} H \sim 2 \mathrm{~T}$ as previously observed and claimed to result from the axial current among Weyl points. (c) Asymmetric or Hall-like component $\rho_{H}=\left[\rho\left(\mu_{0} H\right)-\rho\left(-\mu_{0} H\right)\right] t /\left(2 \mu_{0} H\right)$ as a function of $\mu_{0} H$ where $t$ is the sample thickness. $\rho_{H}$ is nonlinear but saturates beyond $\mu_{0} H \sim 25 \mathrm{~T}$ which is the field beyond which $\rho_{x x}$ increases again as a function of $\mu_{0} H$ while also displaying hysteresis. Both observations point toward an electronic phase transition which probably is also topological in character. (d) $\rho$ as a function of $\mu_{0} H$ for a fourth single crystal (sample 4) also at $T=1.4 \mathrm{~K}$. This sample displays a more pronounced asymmetric magnetoresistivity with respect to sample 3 indicating a larger misalignment of $\mu_{0} H$ with respect to the $a$ axis. (e) and (f) $\rho_{x x}$ and $\rho_{H}$ for sample 4 as functions of $\mu_{0} H$, where the red line [in (e)] is an example of a polynomial fit to the magnetoresistive background. The oscillatory signal $\Delta \rho_{x x}$, superimposed onto $\rho_{x x}$, is obtained by subtracting the polynomial background from the experimental data. (g) Magnetic torque $\boldsymbol{\tau}=\mathbf{M} \times \mu_{0} \mathbf{H}$ as a function of $\mu_{0} \mathbf{H}$ oriented nearly along the $a$ axis under $T=1.4 \mathrm{~K}$. Magenta arrow indicates an anomaly close to the value in a field where the magnetoresistivity crosses from positive $\left[\partial \rho_{x x} / \partial\left(\mu_{0} H\right)>0\right]$ to negative $\left[\partial \rho_{x x} / \partial\left(\mu_{0} H\right)<0\right]$ as the field increases. Blue arrow indicates the onset of hysteresis. The overall behavior of $\tau$, i.e., from negative to positive values upon crossing the quantum limit, is akin to the observations of Ref. [26]. (h) and (i) $\Delta \rho_{x x}$ as a function of $\left(\mu_{0} H\right)^{-1}$ for samples 3 and 4, respectively. The difference in the periods of the oscillatory signals indicates either a small difference in the relative position of the Fermi level(s) between both samples or a small difference in the angular orientation between both samples. The abrupt change in periodicity beyond a certain critical field is indicated by a pink vertical line.

from current jetting: in the first scenario one should observe nearly concentric traces while in the latter one should see a lopsided expansion of the traces resembling a shock wave as seen in elemental $\mathrm{Bi}$ [21] and in $\mathrm{NiTe}_{2}$ [40]. As seen, the traces are concentric indicating, according to Ref. [21], that the negative longitudinal magnetoresistivity observed in TaAs indeed would result from the axial current among its type-I Weyl nodes.

In the Supplemental Material [34], Figs. S2 and S3, we provide a detailed evaluation of the transverse and longitudinal magnetoresistivities as well as the Hall response of TaAs, finding that (i) the transverse magnetoresistivity is far more pronounced at higher temperatures despite the presence of pronounced phonon scattering and becomes pronouncedly asymmetric at low $T$ 's due to a large superimposed Hall component, (ii) the longitudinal negative magnetoresistivity only emerges at temperatures below $T=50 \mathrm{~K}$ displaying also an asymmetry, and (iii) the extracted Hall angle $\Theta_{H}$ saturates at a value as large as $\simeq 82.5^{\circ}$ indicating that the average between positive and negative field sweeps is required to properly extract the magnetoresistivity signal from TaAs, regardless of the configuration of the electrical contacts used. A large superimposed Hall signal can lead to negative readings of the magnetoresistance and be misinterpreted as evidence for current jetting.

Given the previously described large transverse Hall and PHE effects superimposed onto the magnetoresistivity of TaAs, and their interplay with the negative LMR observed below $T=50 \mathrm{~K}$, it is pertinent to ask how the magnetoresistivity might be influenced by the suppression of the Weyl carriers at and beyond the quantum limit, or when one reaches the $n=0$ Landau level.

\section{Suppression of the negative longitudinal magnetoresistivity upon surpassing the quantum limit}

In Fig. 4 we evaluate the LMR, that is $\rho_{x x}\left(\mu_{0} H\right)$ for $\mu_{0} \boldsymbol{H}\|\boldsymbol{j}\| a$-axis, for other two polished TaAs single crystals (samples 3 and 4) having thicknesses approaching $100 \mu \mathrm{m}$, using two pointlike voltage contacts at one edge of these crystals; see the Supplemental Material [34]. This configuration of contacts should lead to a smaller mixing between Hall and magnetoresistivity signals. As mentioned in the Introduction, the application of a component of the magnetic field along an applied electric field is predicted to break the chiral symmetry among Weyl points, creating a net flow of 
charge carriers, or an axial current, from one Weyl point to the other having opposite chirality [13-16]. This axial current is predicted to induce a negative longitudinal magnetoresistivity indeed observed in TaAs [18,19], TaP [42], $\mathrm{Cd}_{3} \mathrm{As}_{2}$ [43], $\mathrm{Na}_{3} \mathrm{Bi}$ [31], and $\mathrm{ZrTe}_{5}$ [44]. In contrast, the large negative LMR observed in TaP was claimed to result from a fieldinduced inhomogeneous current distribution [20], suggesting that this so-called current jetting effect might have contaminated the results in those reports [18,19,31,42-44], although current jetting is claimed to be irrelevant for $\mathrm{Na}_{3} \mathrm{Bi}$ or $\mathrm{GdBiPt}$ while dominating the longitudinal transport in monoarsenides [21].

In Fig. 4(a) we evaluate the longitudinal transport of sample 3 under fields all the way up to $\pm 45 \mathrm{~T}$. Here, great care was taken to align the sample along the basal plane with use of a Hall sensor and through the minimization of the Hall component on the transport data. At very high fields very small misalignments, a fraction of a degree, lead to large transverse Hall and magnetoresistive components superimposed on the data. As in the previously shown measurements and samples, a marked asymmetry is observed between positive (magenta trace) and negative (blue) field sweeps, although these traces were collected from both voltage contacts situated along the same edge of the sample with the intention of evaluating solely its LMR. This asymmetry ought to result from a large superimposed Hall component, i.e., a mixture of the APHE and the transverse Hall signal resulting from the lack of high precision in the angular positioning $\left(\leqslant 0.3^{\circ}\right)$. Therefore, the average between both traces should yield the pure LMR component. As seen in Fig. 4(b), the LMR of sample 3 and for $\mu_{0} H \| a$-axis displays three regions: (i) a low-field region dominated by positive magnetoresistivity observed in all previous studies [18-20,31,42-44] and claimed to result from three-dimensional weak antilocalization [19], (ii) a region characterized by negative LMR previously claimed to result from the axial anomaly, and (iii) a high-field region, whose onset is indicated by the vertical blue arrow, that is characterized by positive and hysteretic magnetoresistivity pointing to a first-order phase transition upon surpassing the quantum limit. The negative LMR is observed from higher field values than those in Ref. [19] likely due to a misalignment between $\mu_{0} H$ and the $a$ axis. Figure 4(c) plots the superimposed Hall component (difference between traces collected under positive and negative fields) normalized by the field indicating that a well-defined Hall constant is observed only beyond the transition. Figures 4(d), 4(e) and 4(f) present similar data for sample 4 . The larger values attained by its raw magnetoresistivity, when compared to sample 3 , indicate a more pronounced sample misalignment. This is also evident from the narrower range in fields where the negative LMR is observed or the larger values of the Hall component due to a sizable transverse Hall signal. Therefore, at low temperatures, all measured crystals display a region in a field where the LMR presents a negative slope, once the Hall component(s) are subtracted, despite the differences in sample dimensions, configuration of contacts used, and even sample alignment.

To provide thermodynamic evidence for the hysteretic phase transition beyond the QL, we also measured the magnetic torque $\vec{\tau}=\mu_{0} \vec{M} \times \vec{H}$ of yet another TaAs single crystal (not shown in Fig. S1) at $T=1.4 \mathrm{~K}$, and as a function of $\mu_{0} H$ along a direction tilted a few degrees with respect to the basal plane. Here, $\vec{M}$ is the sample's magnetization. $\tau$ as a function of $\mu_{0} \mathrm{H}$ is shown in Fig. $4(\mathrm{~g})$ revealing the behavior already described in the Introduction, namely a negative response at low fields, associated with the competition between the diamagnetism and the paramagnetism inherent to the Weyl dispersion, which switches to a purely paramagnetic one as the QL is reached, as previously reported for NbAs [26]. The magnetic torque also reveals mild hysteresis emerging at nearly the same field value, i.e., $\mu_{0} H \sim 25 \mathrm{~T}$, where the negative LMR in sample 3 becomes suppressed. Figures 4(h) and 4(i) plot the oscillatory signal $\Delta \rho_{x x}$ superimposed onto the LMR of samples 3 and 4 , respectively. In these panels we included the frequencies associated with the observed oscillations. We extracted the frequencies directly from their periods instead of taking the Fourier transform due to the very small number of oscillations. Below the quantum limit, both samples display a frequency $F_{\alpha}$ on the order of $(20 \pm 5) \mathrm{T}$, albeit sample 4 also displays a superimposed small frequency $F_{\beta}$ of only $\sim 6 \mathrm{~T}$. The difference in the oscillatory patterns between both samples is attributable to difference in sample quality, relative alignment of the field with respect to the $a$ axis, and perhaps even to a small difference in the relative position of their chemical potential due to small variations in stoichiometry. However, for both samples there is an abrupt change in the sample-dependent oscillatory pattern once the external field surpasses a value in the neighborhood of the quantum limiting $\mu_{0} H_{Q} \sim 20 \mathrm{~T}$ which is slightly higher than the value found for fields along the $c$ axis, or $\sim 16 \mathrm{~T}$. Despite the surprising limitations imposed by a maximum field of $45 \mathrm{~T}$, both samples (see similar data from sample 2 in Fig. S4 of the Supplemental Material [34]) indicate that the oscillatory pattern changes abruptly when the QL limit [45] is surpassed, seemingly revealing much higher frequencies, i.e., ranging between 50 and $150 \mathrm{~T}$. These very few oscillations of very small amplitude prevent us from providing a precise value for the emerging frequency. However, the apparent emergence of a larger periodicity, implying larger Fermi surface sheets, would be surprising and difficult to reconcile with the behavior of the torque indicating, according to Ref. [26], that only the $n=0$ Landau would remain beyond $\mu_{0} H_{Q}$. Therefore, the magnetic torque would imply that these "oscillations" in the resistivity involve quasiparticles at fractional filling factors naturally explaining why they deviate from the Landau index plot in Fig. S4(d) [34]. A similar scenario was proposed for for $\mathrm{ZrTe}_{5}$ upon surpassing the QL [46]. For comparison, see transport data for NbAs [47]. Notice that the abrupt change in periodicity observed above the quantum limiting field cannot be attributed to the Zeeman effect since this effect, which is linear in field, produces a relatively small deformation of the Fermi surface below $\mu_{0} H_{Q}$. In the discussion associated to Fig. S4 we evaluate the Berry phase through different methods and field windows finding different values, although it is known that quantum oscillations cannot extract the correct value for the Berry phase in three-dimensional systems [48,49]. These oscillations cannot be attributed to simple magnetic breakdown either given that the original periodicities seen at lower fields completely disappear at the transition (instead, both frequencies should coexist). 
Recently, an electronic phase transition was observed in TaAs for fields (in excess of $\mu_{0} H=60 \mathrm{~T}$ ) and currents applied along its $c$ axis [50]. It is characterized by a $\sim 2$ orders of magnitude increase in magnetoresistivity and claimed to result from the opening of a gap at the Weyl nodes [50]. Our results, namely the abrupt change in the quantum oscillatory pattern, the suppression of negative LMR for fields along the $a$ axis, and the observation of hysteresis in transport and in thermodynamic quantities, indicate that the Weyl dispersion is already affected by much lower fields. The observation of hysteresis does indicate a first-order phase transition toward a field-induced phase perhaps akin to those observed in graphite [30], or in quasi-one-dimensional organic systems [51,52]. It might correspond to a magnetic-field-induced density wave state having for modulation the wave vector connecting both Weyl nodes of opposite chiralities as predicted for pyrochlore iridates [53]. The field-induced opening of a gap was indeed predicted for the monoarsenides based on ab initio calculations due to the mixing of the zeroth Landau levels associated with the Weyl points of opposite chirality [54]. This effect would be rather anisotropic, occurring at much lower fields when these are aligned along the $a$ axis. This contrasts with our observations indicating an abrupt change in the oscillatory pattern for both field orientations and at considerably lower fields than those reported in Refs. [50] and [54]. In Fig. S5 of the Supplemental Material [34], we include positive magnetoresistivity data for sample 5 revealing the strongly hysteretic transition and the purely hysteretic signal from sample 3 suggesting the possibility of multiple phase transitions.

In Fig. S6 of the Supplemental Material [34] we include Hall-effect measurements up to $\sim 63 \mathrm{~T}$ applied along the $c$ axis for another TaAs single crystal, indicating a decrease in the slope of the transverse Hall resistivity $\rho_{x y}\left(\mu_{0} H\right)$ upon surpassing the quantum limit. This could be interpreted as either (i) an increase in carrier density which would be at odds with the disappearance of the small frequencies seen in the Shubnikov-de Haas signal or (ii) the loss of the high-mobility Weyl electrons originally located at the Fermi level. For a high-mobility semimetal like TaAs, one would expect the loss of carriers to lead to poorer carrier compensation and therefore to a more pronounced slope in the bare Hall signal. The opposite is observed, and therefore we conclude that this transition leads to an overall decrease in carrier mobility perhaps due to the gapping of the Fermi surfaces located on the Weyl dispersing bands. This same Hall signal, collected under pulsed fields, also shows another change in slope around $\mu_{0} H \simeq 50 \mathrm{~T}$ which coincides with the transition reported by Ref. [50]. If the first change in slope was attributable to the loss of one set of Weyl electrons (e.g., W1), this second transition might be attributable to either the loss of the second set of Weyl carriers (at the W2 points) or the loss of the conventional holes (see Fig. 1(a) in Ref. [50]). Our results present certain similarities with those in Ref. [55] which in TaP show a series of anomalies in the magnetotransport properties below and above the quantum limit where the Hall effect is observed to change sign abruptly. This was attributed to the gapping of the Weyl nodes resulting from the competition between the magnetic wave vector and the separation in $k$ space of the W1 nodes. Here, the important point is that our study

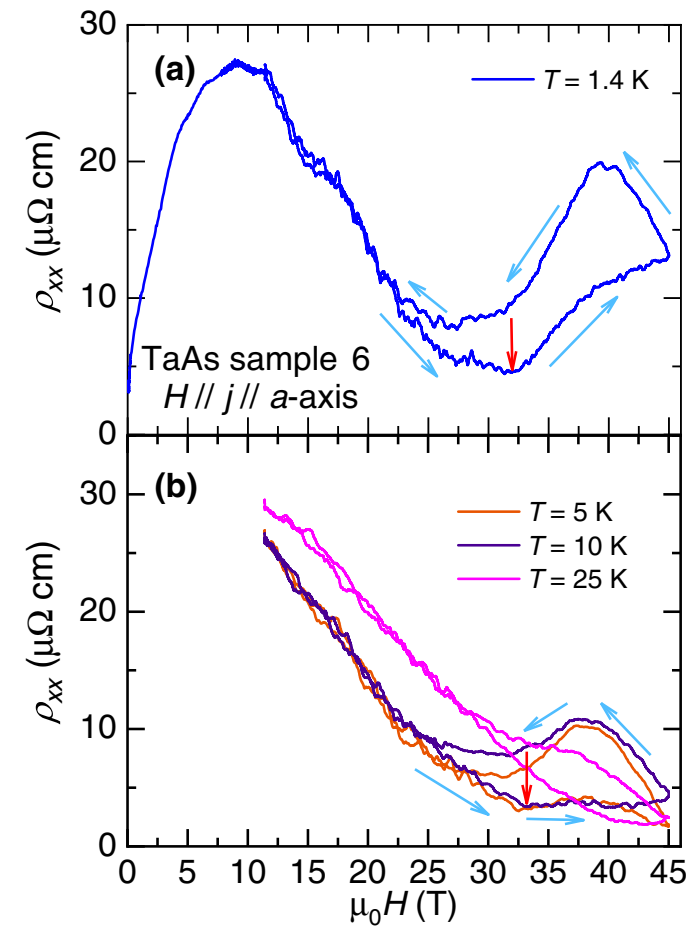

FIG. 5. Hysteresis in the magnetoresistivity at the field-induced phase transition and recovery of positive longitudinal magnetoresistivity. (a) Nonsymmetrized $\rho_{x x}$ for a sixth TaAs crystal as a function of $\mu_{0} H$ applied nearly along the electrical current $j$ injected along the $a$ axis of the crystal at a temperature $T=1.4 \mathrm{~K}$. Its initial positive slope becomes negative beyond $H \sim 8 \mathrm{~T}$, an effect ascribed to the axial anomaly among Weyl points. For fields above $H_{c} \simeq 32.5 \mathrm{~T}$ (indicated by the red arrow), the negative slope becomes positive again producing a large hysteresis as the field is swept to lower values. This change in slope combined with the hysteresis indicates a magnetic-field-induced first-order phase transition. $H_{c}$ is close in value to the frequency $F$ of the superimposed weak oscillatory signal, or $F=(30 \pm 5) \mathrm{T}$, indicating that it occurs in the neighborhood of the quantum limit. (b) $\rho_{x x}$ as a function of $H \| a$-axis for three temperatures. The hysteresis survives under temperatures as high as $25 \mathrm{~K}$.

provides evidence for two electronic phase transitions. Our results indicate that the one occurring at the $\mathrm{QL}$ involves the suppression of the Weyl carriers, and therefore it ought to be topological in nature.

Finally, Fig. 5(a) displays the nonsymmetrized $\rho_{x x}\left(\mu_{0} H\right)$ for $\mu_{0} \boldsymbol{H}$ nearly along $\boldsymbol{j} \| \boldsymbol{a}$-axis, and for yet a sixth TaAs single crystal. Blue arrows indicate both field increasing and decreasing sweeps. The SdH oscillations observed in the background of the magnetoresistivity yield a frequency $F \sim(30 \pm 5) \mathrm{T}$ which essentially coincides with $H_{\text {kink }}$ (indicated by red arrow), where the slope of the magnetoresistivity changes from negative to positive and which, combined with the pronounced hysteresis, indicates a first-order transition upon reaching the quantum limit. Hysteresis and its increase at lower temperatures could result from the existence of density-wave-like domains having the size of their coherence length. Domain boundaries would scatter the charge carriers thus increasing the resistivity. At higher temperatures, 
thermal activation processes would promote a fraction of these carriers across the domain boundaries thus leading to a smaller hysteresis. Figure 5(b) displays the longitudinal magnetoresistivity as a function of $\mu_{0} H$ for three temperatures. Notice the disappearance of the sharp kink at $H_{\text {kink }}$ and of the concomitant hysteresis upon increasing the temperature which indicates that this phase-transition emerges upon decreasing $T$. Therefore, Figs. 4 and 5 or samples 3, 4, and 6 indicate quite clearly that the negative LMR observed in TaAs correlates with its zero magnetic field electronic structure at the Fermi level. For fields along the $a$ axis, TaAs undergoes a first-order electronic phase-transition upon approaching the quantum limit, which might involve the gapping of the Weyl nodes and which is followed by features at fractional filling factors as the field is increased.

As for the hysteresis one could assume a certain analogy with graphite where the external magnetic field is believed to induce a state akin to a charge-density wave [30]. These electronic modulations become pinned by defects, and are characterized by domains having the size of a certain coherence length. At higher temperatures thermal activation can both displace the domain boundaries and help to locally unpin the density wave. It can also promote carriers across their boundaries. These processes are reduced as the temperature is lowered thus favoring hysteresis. For a field-induced transition one expects the coherence length associated with the density wave modulation to be field dependent, implying that the size and the configuration of the domains evolve with field, leading to hysteresis as the charge carriers interact with the domain boundaries. This effect should decrease in amplitude as thermal activation comes into play while the hysteresis should be history dependent.

At this point, we must mention that several recent papers have reanalyzed the electrical transport properties of systems characterized by pronounced Berry curvature, e.g., metals and topological insulators, through semiclassical transport equations finding a new contribution that leads to negative longitudinal magnetoresistivity in absence of the axial anomaly [56-58]. This effect would be observable in the absence of the band crossings that lead, for example, to the Weyl nodes or other nontrivial topological singularities in the bulk. However, this semiclassical approach which is adequate to low magnetic fields makes these models inadequate to describe the negative magnetoresistivity observed in TaAs in the vicinity of the quantum limit. In $\mathrm{Cd}_{3} \mathrm{As}_{2}$ this effect was ascribed to conductivity fluctuations [60] which are expected to become pronounced in systems displaying pronounced differences in the relative mobility and/or in charge-carrier densities between the different types of charge carriers. In thin films of $\mathrm{Cd}_{3} \mathrm{As}_{2}$ this difference becomes pronounced at higher temperatures precisely where one observes a pronounced increase in the magnetoconductivity [60]. Nevertheless, according to the two-band analysis in Ref. [18], TaAs would display very pronounced differences in electron and hole mobilities over the entire temperature range, with the most pronounced differences in carrier density observed upon approaching room temperature. Nonetheless, we have shown here that negative magnetoresistivity emerges in TaAs well below $T=100 \mathrm{~K}$, which is at odds with the conductance fluctuations scenario. Finally, Ref. [59] observes a large but distorted PHE-like signal in TaP, i.e., nonsinusoidal or displaying a markedly distorted triangular signal, and attributed its behavior as well as the angular dependence of the magnetoresistivity to the anisotropy of the orbital magnetoresistivity. Nevertheless, it is impossible to compare our data set with the one in Ref. [59] where the authors admit a strong role for current jetting, given that the tests performed by us in our samples indicate that our TaAs data are not contaminated by this effect. Therefore, it is not surprising that we end up reaching diametrically opposed conclusions.

Perhaps a more intriguing scenario is the one presented by Ref. [61]. It analyzes the magnetoconductivity of massless and massive Dirac fermions subjected to quantum interference induced by scalar impurity scattering potentials, finding a crossover from positive to negative magnetoresistivity associated with a strong competition between weak localization and weak antilocalization. In this scenario, the recovery of positive magnetoresistivity upon surpassing the quantum limit, as seen by us, might be ascribable to the suppression of the Weyl fermions. Nevertheless, this model does not address the very pronounced Hall angle, the large PHE, or the evidence for the APHE exposed here.

\section{SUMMARY}

The ensemble of anomalous transport properties shown here, namely an anomalously large Hall angle for a threedimensional system, a large planar Hall effect, and a negative slope for the longitudinal magnetoresisivity emerging only below $\sim 50 \mathrm{~K}$, suggests a unique texture of the Berry phase and points to the prominent role played by the axial/chiral anomaly in TaAs. Experimental procedures suggested by Ref. [21] to evaluate the role of current jetting in our samples provides no evidence for it. Instead, our study indicates that conventional current contacts do not lead, in our polished crystals, to the extremely inhomogeneous current distributions reported in Refs. [20,21] associated with very small pointlike current contacts, and that was claimed to indicate that current jetting dominates the electrical transport in monoarsenides. Instead, we observe a very large Hall signal superimposed on the magnetoresistivity, regardless of the configuration of the contacts used. If not taken into consideration, or properly subtracted, it could be mistaken for evidence for current jetting when the electrical contacts are placed at different locations throughout the crystal. Therefore, we are led to conclude that the effects observed here are most likely intrinsic to TaAs, and associated with the unique texture of its Berry phase and possibly with the chiral anomaly among its Weyl nodes. We have discussed recently proposed alternative magnetoresistivity scenarios [56-60], concluding that they cannot explain the totality of our observations.

Negative longitudinal magnetoresistivity [18,19,21,31,4244] and a large planar Hall effect have been predicted theoretically $[24,25]$ and observed experimentally in several compounds $[37,38]$. From an experimental perspective, it would be important to clarify whether the PHE effect observed here disappears under fields beyond the quantum limit which seemingly gap the Weyl nodes. This would unambiguously connect the nodes to the observation of this effect. 
The observation of a hysteretic phase transition that leads to an abrupt change in the quantum oscillatory pattern confirms that the electronic structure of TaAs undergoes an electronic and likely topological phase transition(s) upon approaching and surpassing the quantum limit. The negative longitudinal magnetoresistivity, initially attributed to the axial anomaly among Weyl nodes, is suppressed at the transition suggesting the suppression of the Weyl nodes. Notice that the disappearance of the longitudinal negative magnetoresistivity at the transition might be considered evidence for the suppression of the Weyl nodes even in the current jetting scenario, since it would be attributable to a decrease in carrier mobility. Notice that lower carrier mobilities are consistent with our Hall-effect measurements that reveal a smaller Hall constant emerging beyond the quantum limiting field. For fields along the $c$ axis the Hall effect reveals a second decrease in slope at the onset field where an abrupt phase transition was previously reported [50], thus indicating that this system might undergo two subsequent field-induced phase transitions as the field is increased. Remarkably, beyond the quantum limit and for both field orientations we still observe quasiperiodic oscillations. Given that the overall response of the magnetic torque as a function of the field suggests, according to Ref. [26], that only the $n=0$ Landau level should survive the crossing of the quantum limiting field, one cannot but conclude that these features should involve quasiparticles at fractional filling factors.

These observations lead to a series of questions given that TaAs displays two sets of Weyl nodes characterized by distinct separations in $k$ space [3]. Are both sets of Weyl nodes gapped at the first hysteretic phase transition? Does this transition become second-order-like for fields along the $c$ axis? What would be the origin of the second phase transition seen at higher fields? The transition reported in Ref. [50] upon approaching and surpassing the quantum limit was claimed to result from the competition between the magnetic wavelength and the wave vector(s) connecting Weyl points of opposite chirality [55]. Such a competition would be akin to what is observed in bulk quasi-one-dimensional organic conductors that undergo a cascade of first-order, magnetic-field-induced density wave transitions that display a concomitant quantum Hall effect [51,52]. Therefore, if each one of these transitions involved the gapping of one set of Weyl nodes, why is the negative longitudinal magnetoresistivity suppressed already at the first transition, regardless of the mechanism leading to it (the second set of nodes should survive the quantum limiting field)? Finally, what is the origin of the quasioscillatory features observed beyond the quantum limit?

We hope that our work stimulates the quest for the respective answers.

\section{ACKNOWLEDGMENTS}

L.B. is supported by the US Department of Energy, Basic Energy Sciences program through Award No. DESC0002613. Work at Princeton University was supported by the Gordon and Betty Moore Foundations Emergent Phenomena in Quantum Systems Initiative through Grant No. GBMF4547 (M.Z.H.). F.C. acknowledges the support provided by MOST-Taiwan under Project No. 102-2119-M-002004. J.Y.C. acknowledges support from NSF DMR-1700030. The NHMFL acknowledges NSF support through DMR1157490 and DMR-1644779, and the State of Florida. The data that support the findings of this study are available from the corresponding authors upon reasonable request.
[1] A. M. Turner and A. Vishwanath, Beyond Band Insulators: Topology of Semimetals and Interacting Phases (Elsevier, New York, 2013).

[2] S. M. Huang, S. Y. Xu, I. Belopolski, C.-C. Lee, G. Chang, B. Wang, N. Alidoust, G. Bian, M. Neupane, C. Zhang, S. Jia, A. Bansil, L. Hsin and M. Z. Hasan, A Weyl fermion semimetal with surface Fermi arcs in the transition metal monopnictide TaAs class, Nat. Commun. 6, 7373 (2015).

[3] H. Weng, C. Fang, Z. Fang, B. A. Bernevig, and X. Dai, Weyl Semimetal Phase in Noncentrosymmetric TransitionMetal Monophosphides, Phys. Rev. X 5, 011029 (2015).

[4] S. Jia, S.-Y. Xu, and M. Z. Hasan, Weyl semimetals, Fermi arcs and chiral anomalies, Nat. Mater. 15, 1140 (2016).

[5] H. Weyl, Electron and gravitation, Z. Phys. 56, 330 (1929).

[6] P. A. M. Dirac, The quantum theory of electron, Proc. R. Soc. London A 117, 610 (1928).

[7] S.-Y. Xu, N. Alidoust, I. Belopolski, Z. Yuan, G. Bian, T. R. Chang, H. Zheng, V. N. Strocov, D. S. Sanchez, G. Chang, C. Zhang, D. Mou, Y. Wu, L. Huang, C.-C. Lee, S.-M. Huang, B. Wang, A. Bansil, H.-T. Jeng, T. Neupert, A. Kaminski, H. Lin, S. Jia and M. Z. Hasan, Discovery of a Weyl fermion state with Fermi arcs in niobium arsenide, Nat. Phys. 11, 748 (2015).

[8] B. Q. Lv, H. M. Weng, B. B. Fu, X. P. Wang, H. Miao, J. Ma,
P. Richard, X. C. Huang, L. X. Zhao, G. F. Chen, Z. Fang, X. Dai, T. Qian, and H. Ding, Experimental Discovery of Weyl Semimetal TaAs, Phys. Rev. X 5, 031013 (2015).

[9] S.-Y. Xu, I. Belopolski, N. Alidoust, M. Neupane, G. Bian, C. Zhang, R. Sankar, G. Chang, Z. Yuan, C.-C. Lee, S.-M. Huang, H. Zheng, J. Ma, D. S. Sanchez, B. Wang, A. Bansil, F. Chou, P. P. Shibayev, H. Lin, S. Jia, and M. Z. Hasan, Discovery of a Weyl fermion semimetal and topological Fermi arcs, Science 349, 613 (2015).

[10] B. Q. Lv, N. Xu, H. M. Weng, J. Z. Ma, P. Richard, X. C. Huang, L. X. Zhao, G. F. Chen, C. Matt, F. Bisti, V. Strokov, J. Mesot, Z. Fang, X. Dai, T. Qian, M. Shi, and H. Ding, Observation of Weyl nodes in TaAs, Nat. Phys. 11, 724 (2015).

[11] L. X. Yang, Z. K. Liu, Y. Sun, H. Peng, H. F. Yang, T. Zhang, B. Zhou, Y. Zhang, Y. F. Guo, M. Rahn, D. Prabhakaran, Z. Hussain, S.-K. Mo, C. Felser, B. Yan and Y. L. Chen, Weyl semimetal phase in the non-centrosymmetric compound TaAs, Nat. Phys. 11, 728 (2015).

[12] Z. K. Liu, L. X. Yang, Y. Sun, T. Zhang, H. Peng, H. F. Yang, C. Chen, Y. Zhang, Y. F. Guo, D. Prabhakaran, M. Schmidt, Z. Hussain, S.-K. Mo, C. Felser, B. Yan, and Y. L. Chen, Evolution of the Fermi surface of Weyl semimetals in the transition metal pnictide family, Nat. Mater. 15, 27 (2015). 
[13] S. Adler, Axial-vector vertex in spinor electrodynamics, Phys. Rev. 177, 2426 (1969).

[14] J. S. Bell and R. A. Jackiw, A PCAC puzzle: $\pi_{0} \rightarrow \gamma \gamma$ in the $\sigma$-model, Nuovo Cimento A 60, 47 (1969).

[15] H. B. Nielsen and M. Ninomiya, The Adler-Bell-Jackiw anomaly and Weyl fermions in a crystal, Phys. Lett. B 130, 389 (1983).

[16] P. Goswami, J. H. Pixley, and S. Das Sarma, Axial anomaly and longitudinal magnetoresistance of a generic three-dimensional metal, Phys. Rev. B 92, 075205 (2015).

[17] C.-L. Zhang, B. Tong, Z. Yuan, Z. Lin, J. Wang, J. Zhang, C.-Y. Xi, Z. Wang, S. Jia, and C. Zhang, Signature of chiral fermion instability in the Weyl semimetal TaAs above the quantum limit, Phys. Rev. B 94, 205120 (2016).

[18] X. C. Huang, L. Zhao, Y. Long, P. Wang, D. Chen, Z. Yang, H. Liang, M. Xue, H. Weng, Z. Fang, X. Dai, and G. Chen, Observation of the Chiral-Anomaly-Induced Negative Magnetoresistance in 3D Weyl Semimetal TaAs, Phys. Rev. X 5, 031023 (2015).

[19] C.-L. Zhang, S.-Y. Xu, I. Belopolski, Z. Yuan, Z. Lin, B. Tong, G. Bian, N. Alidoust, C.-C. Lee, S.-M. Huang, T.-R. Chang, G. Chang, C.-H. Hsu, H.-T. Jeng, M. Neupane, D. S. Sanchez, H. Zheng, J. Wang, H. Lin, C. Zhang, H.-Z. Lu, S.-Q. Shen, T. Neupert, M. Z. Hasan, and S. Jia, Signatures of the AdlerBell-Jackiw chiral anomaly in a Weyl fermion semimetal, Nat. Commun. 7, 10735 (2016).

[20] F. Arnold, C. Shekhar, S.-C. Wu, Y. Sun, R. D. dos Reis, N. Kumar, M. Naumann, M. O. Ajeesh, M. Schmidt, A. G. Grushin, J. H. Bardarson, M. Baenitz, D. Sokolov, H. Borrmann, M. Nicklas, C. Felser, E. Hassinger and B. Yan, Negative magnetoresistance without well-defined chirality in the Weyl semimetal TaP, Nat. Commun. 7, 11615 (2016).

[21] S. H. Liang, J. J. Lin, S. Kushwaha, J. Xing, N. Ni, R. J. Cava, and N. P. Ong, Experimental Tests of the Chiral Anomaly Magnetoresistance in the Dirac-Weyl Semimetals $\mathrm{Na}_{3} \mathrm{Bi}$ and GdPtBi, Phys. Rev. X 8, 031002 (2018).

[22] Y. Baum, E. Berg, S. A. Parameswaran, and A. Stern, Current at a Distance and Resonant Transparency in Weyl Semimetals, Phys. Rev. X 5, 041046 (2015).

[23] P. J. W. Moll, N. L. Nair, T. Helm, A. C. Potter, I. Kimchi, A. Vishwanath, and J. G. Analytis, Transport evidence for Fermiarc-mediated chirality transfer in the Dirac semimetal $\mathrm{Cd}_{3} \mathrm{As}_{2}$, Nature (London) 535, 266 (2016).

[24] A. A. Burkov, Giant planar Hall effect in topological metals, Phys. Rev. B 96, 041110(R) (2017).

[25] S. Nandy, G. Sharma, A. Taraphder, and S. Tewari, Chiral Anomaly as the Origin of the Planar Hall Effect in Weyl Semimetals, Phys. Rev. Lett. 119, 176804 (2017).

[26] P. J. W. Moll, A. C. Potter, N. L. Nair, B. J. Ramshaw, K. A. Modic, S. Riggs, B. Zeng, N. J. Ghimire, E. D. Bauer, R. Kealhofer, F. Ronning, and J. G. Analytis, Magnetic torque anomaly in the quantum limit of Weyl semimetals, Nat. Commun. 7, 12492 (2016).

[27] F. Arnold, M. Naumann, S.-C. Wu, Y. Sun, M. Schmidt, H. Borrmann, C. Felser, B. Yan, and E. Hassinger, Chiral Weyl Pockets and Fermi Surface Topology of the Weyl Semimetal TaAs, Phys. Rev. Lett. 117, 146401 (2016).

[28] C.-C. Lee, S.-Y. Xu, S.-M. Huang, D. S. Sanchez, I. Belopolski, G. Chang, G. Bian, N. Alidoust, H. Zheng, M. Neupane,
B. Wang, A. Bansil, M. Z. Hasan, and H. Lin, Fermi surface interconnectivity and topology in Weyl fermion semimetals TaAs, TaP, NbAs, and NbP, Phys. Rev. B 92, 235104 (2015).

[29] Y. Luo, N. J. Ghimire, M. Wartenbe, H. Choi, M. Neupane, R. D. McDonald, E. D. Bauer, J. Zhu, J. D. Thompson, and F. Ronning, Electron-hole compensation effect between topologically trivial electrons and nontrivial holes in NbAs, Phys. Rev. B 92, 205134 (2015).

[30] B. Fauqué, D. LeBoeuf, B. Vignolle, M. Nardone, C. Proust, and K. Behnia, Two Phase Transitions Induced by a Magnetic Field in Graphite, Phys. Rev. Lett. 110, 266601 (2013).

[31] J. Xiong, S. K. Kushwaha, T. Liang, J. W. Krizan, M. Hirschberger, W. Wang, R. J. Cava, and N. P. Ong, Evidence for the chiral anomaly in the Dirac semimetal $\mathrm{Na}_{3} \mathrm{Bi}$, Science 350, 413 (2015).

[32] T. Besara, D. A. Rhodes, K.-W. Chen, S. Das, Q. R. Zhang, J. Sun, B. Zeng, Y. Xin, L. Balicas, R. E. Baumbach, E. Manousakis, D. J. Singh, and T. Siegrist, Coexistence of Weyl physics and planar defects in the semimetals TaP and TaAs, Phys. Rev. B 93, 245152 (2016).

[33] K. Yoshida, Transport of spatially inhomogeneous current in a compensated metal under magnetic fields. III. A case of bismuth in longitudinal and transverse magnetic fields, J. Appl. Phys. 51, 4226 (1980).

[34] See Supplemental Material at http://link.aps.org/supplemental/ 10.1103/PhysRevB.100.115138 for pictures of several of the measured single crystals, evaluation of transverse and longitudinal magnetoresistivity, evaluation of the Hall effect leading to a very large Hall angle, evolution of the electronic structure for fields along the $c$ axis, upon surpassing the quantum limit, hysteresis for another sample slightly misaligned with respect to the $c$ axis, and the Hall effect collected under pulsed fields indicating two field-induced transitions.

[35] W. A. Reed, E. I. Blount, J. A. Marcus, and A. J. Arko, Anomalous longitudinal magnetoresistance in metals, J. Appl. Phys. 42, 5453 (1971).

[36] B. Pippard, Longitudinal magnetoresistance, Proc. R. Soc. A 282, 464 (1964).

[37] N. Kumar, S. N. Guin, C. Felser, and C. Shekhar, Planar Hall effect in the Weyl semimetal GdPtBi, Phys. Rev. B 98, 041103(R) (2018).

[38] H. Li, H.-W. Wang, H. He, J. Wang, and S.-Q. Shen, Giant anisotropic magnetoresistance and planar Hall effect in the Dirac semimetal $\mathrm{Cd}_{3} \mathrm{As}_{2}$, Phys. Rev. B 97, 201110(R) (2018).

[39] D. D. Liang, Y. J. Wang, W. L. Zhen, J. Yang, S. R. Weng, X. Yan, Y. Y. Han, W. Tong, W. K. Zhu, L. Pi, and C. J. Zhang, Origin of planar Hall effect in type-II Weyl semimetal $\mathrm{MoTe}_{2}$, AIP Adv. 9, 055015 (2019).

[40] Q. Liu, F. Fei, B. Chen, X. Bo, B. Wei, S. Zhang, M. Zhang, F. Xie, M. Naveed, X. Wan, F. Song, and B. Wang, Nontopological origin of the planar Hall effect in the type-II Dirac semimetal $\mathrm{NiTe}_{2}$, Phys. Rev. B 99, 155119 (2019).

[41] T. Liang, J. Lin, Q. Gibson, S. Kushwaha, M. Liu, W. Wang, H. Xiong, J. A. Sobota, M. Hashimoto, P. S. Kirchmann, Z.-X. Shen, R. J. Cava, and N. P. Ong, Anomalous Hall effect in $\mathrm{ZrTe}_{5}$, Nat. Phys. 14, 451 (2018).

[42] J.-H. Du, H.-D. Wang, Q. Chen, Q.-H. Mao, R. Khan, B.-J. $\mathrm{Xu}$, Y.-X. Zhou, Y.-N. Zhang, J.-H. Yang, B. Chen, C.-M. 
Feng, and M.-H. Fang, Large unsaturated positive and negative magnetoresistance in Weyl semimetal TaP, Sci. China-Phys. Mech. Astron. 59, 657406 (2016).

[43] H. Li, H. He, H.-Z. Lu, H. Zhang, H. Liu, R. Ma, Z. Fan, S.-Q. Shen, and J. Wang, Negative magnetoresistance in Dirac semimetal $\mathrm{Cd}_{3} \mathrm{As}_{2}$, Nat. Commun. 7, 10301 (2016).

[44] Q. Li, D. E. Kharzeev, C. Zhang, Y. Huang, I. Pletikosić, A. V. Fedorov, R. D. Zhong, J. A. Schneeloch, G. D. Gu, and T. Valla, Chiral magnetic effect in $\mathrm{ZrTe}_{5}$, Nat. Phys. 12, 550 (2016).

[45] D. Shoenberg, Magnetic Oscillations in Metals (Cambridge University Press, New York, 2011).

[46] W. Yu, Y. Jiang, J. Yang, Z. L. Dun, H. D. Zhou, Z. Jiang, P. $\mathrm{Lu}$, and W. Pan, Quantum oscillations at integer and fractional Landau level indices in single-crystalline $\mathrm{ZrTe}_{5}$, Sci. Rep. 6, 35357 (2016).

[47] N. J. Ghimire, Y. K. Luo, M. Neupane, D. J. Williams, E. D. Bauer, and F. Ronning, Magnetotransport of single crystalline NbAs, J. Phys.: Condens. Matter 27, 152201 (2015).

[48] C. M. Wang, H.-Z. Lu, and S.-Q. Shen, Anomalous Phase Shift of Quantum Oscillations in 3D Topological Semimetals, Phys. Rev. Lett. 117, 077201 (2016).

[49] K.-W. Chen, X. Lian, Y. Lai, N. Aryal, Y.-C. Chiu, W. Lan, D. Graf, E. Manousakis, R. E. Baumbach, and L. Balicas, Bulk Fermi Surfaces of the Dirac Type-II Semimetallic Candidates $\mathrm{MAl}_{3}$ (Where $M=\mathrm{V}, \mathrm{Nb}$, and Ta), Phys. Rev. Lett. 120, 206401 (2018).

[50] B. J. Ramshaw, K. A. Modic, A. Shekhter, Yi Zhang, E.-A. Kim, P. J. W. Moll, M. D. Bachmann, M. K. Chan, J. B. Betts, F. Balakirev, A. Migliori, N. J. Ghimire, E. D. Bauer, F. Ronning, and R. D. McDonald, Quantum limit transport and destruction of the Weyl nodes in TaAs, Nat. Commun. 9, 2217 (2018).

[51] S. T. Hannahs, J. S. Brooks, W. Kang, L. Y. Chiang, and P. M. Chaikin, Quantum Hall Effect in a Bulk Crystal, Phys. Rev. Lett. 63, 1988 (1989).
[52] J. R. Cooper, W. Kang, P. Auban, G. Montambaux, D. Jerome, and K. Bechgaard, Quantized Hall Effect and a New Field-Induced Phase Transition in the Organic Superconductor (TMTSF) $)_{2} \mathrm{PF}_{6}$, Phys. Rev. Lett. 63, 1984 (1989).

[53] K. Y. Yang, Y. M. Lu, and Y. Ran, Quantum Hall effects in a Weyl semimetal: Possible application in pyrochlore iridates, Phys. Rev. B 84, 075129 (2011).

[54] P. Kim, J. H. Ryoo, and C. H. Park, Breakdown of the Chiral Anomaly in Weyl Semimetals in a Strong Magnetic Field, Phys Rev. Lett. 119, 266401 (2017).

[55] C.-L. Zhang, S.-Y. Xu, C. M. Wang, Z. Lin, Z. Z. Du, C. Guo, C.-C. Lee, H. Lu, Y. Feng, S.-M. Huang, G. Chang, C.-H. Hsu, H. Liu, H. Lin, L. Li, C. Zhang, J. Zhang, X.-C. Xie, T. Neupert, M. Z. Hasan, H.-Z. Lu, J. Wang, and S. Jia, Magnetictunnelling-induced Weyl node annihilation in TaP, Nat. Phys. 13, 979 (2017).

[56] Y. Gao, S. A. Yang, and Q. Niu, Intrinsic relative magnetoconductivity of nonmagnetic metals, Phys. Rev. B 95, 165135 (2017).

[57] X. Dai, Z. Z. Du, and H.-Z. Lu, Negative Magnetoresistance without Chiral Anomaly in Topological Insulators, Phys. Rev. Lett. 119, 166601 (2017).

[58] H.-P. Sun and H.-Z. Lu, Quantum transport in topological semimetals under magnetic fields (II), Front. Phys. 14, 33405 (2019).

[59] J. Yang, W. L. Zhen, D. D. Liang, Y. J. Wang, X. Yan, S. R. Weng, J. R. Wang, W. Tong, L. Pi, W. K. Zhu, and C. J. Zhang, Current jetting distorted planar Hall effect in a Weyl semimetal with ultrahigh mobility, Phys. Rev. Mater. 3, 014201 (2019).

[60] T. Schumann, M. Goyal, D. A. Kealhofer, and S. Stemmer, Negative magnetoresistance due to conductivity fluctuations in films of the topological semimetal $\mathrm{Cd}_{3} \mathrm{As}_{2}$, Phys. Rev. B 95, 241113(R) (2017).

[61] B. Fu, H.-W. Wang, and S.-Q. Shen, Quantum Interference Theory of Magnetoresistance in Dirac Materials, Phys. Rev. Lett. 122, 246601 (2019). 